\title{
ON CONTINUOUSLY SEMIMETRIZABLE AND STRATIFIABLE SPACES
}

\author{
CARLOS J. R. BORGES ${ }^{1}$
}

1. Introduction. Recently Professor J. Nagata asked us a question which we will phrase as follows: Is it true that a space $X$ is a Nagata space (i.e. stratifiable ${ }^{2}$ and first countable) if and only if $X$ is $i$-continuously semimetrizable ${ }^{3}$ (see Definition 2.1)? Unfortunately, it turns out that neither implication is valid even for regular cosmic ${ }^{4}$ (hence paracompact) spaces. The situation is not improved by replacing " $i$-continuousiy" by "continuously" in the above question. Nevertheless, it should not be forgotten that every Nagata space is semimetrizable (see Theorem 6.2 of Ceder [2]).

Throughout we use the terminology of Kelley [6].

2. Theorems and proofs. Before answering Nagata's question we establish a few preliminaries.

Definition 2.1. Let $f: X \times Y \rightarrow Z$ be a function, where $X, Y$ and $Z$ are topological spaces. Then $f$ is said to be $i$-continuous $(i=1,2)$ if $f$ is continuous with respect to the $i$ th variable.

Lemma 2.2. Let $X$ be a semimetrizable space and $d$ be a semimetric on $X$ which generates the topology on $X$. Then $d$ is 1-continuous if and only if, for each $\left(p, p^{\prime}\right) \in X \times X$ and sequence $\left\{x_{n}\right\}_{n=1}^{\infty}$ converging to $p$, we get that $\lim _{n} d\left(x_{n}, p^{\prime}\right)=d\left(p, p^{\prime}\right)$.

Proof. Straightforward, since $X$ is clearly first countable.

Example 2.3. There exists a cosmic (see footnote 4) Nagata space $X$ which is not 1-continuously semimetrizable.

Proof. We actually use a space first constructed by Heath [4]. Let $X$ be the set of all points of the plane with a base for a topology $\tau$

Received by the editors September 29, 1967.

1 This research was supported by NSF Grant GP-6390.

2 A $T_{1}$-space $X$ is stratifiable if to each open $U \subset X$ one can assign a sequence $\left\{U_{n}\right\}_{n-1}^{\infty}$ of open subsets of $X$ such that, for all $n, U_{n} \subset U, U_{n} \subset V_{n}$ whenever $U \subset V$, and $\bigcup_{n-1}^{\infty} U_{n}=U$. The correspondence $U \rightarrow\left\{U_{n}\right\}_{n-1}^{\infty}$ is called a stratification of $X$. Stratifiable spaces are the same as the $M_{3}$-spaces of Ceder [2].

'A $T_{1}$-space $X$ is semimetrizable if there exists a real-valued function $d$ on $X \times X$ such that (a) $d(x, y)=d(y, x)$, (b) $d(x, y)=0$ if and only if $x=y$, and (c) a point $p \in X$ is in the closure of a subset $B$ of $X$ if and only if $\inf (\{d(p, b) \mid b \in B\})=0$ (i.e. $d$ generates the topology of $X$ ). The function $d$ is called a semimetric. $X$ is $i$-continuously semimetrizable if $d$ is $i$-continuous.

${ }^{4} \mathrm{~A}$ cosmic space is any $T_{1}$-regular space which is the continuous image of a separable metric space. 
consisting of (1) all open discs and (2) all "butterfly regions" centered on irrational points of the $x$-axis (a "butterfly region" centered at the point $x$ is $\{y \in X|| x-y \mid+a(x, y)<c\}$, where $|x-y|$ is the cartesian distance between $x$ and $y$ and $a(x, y)$ is the smallest nonnegative angle, expressed in radians, formed by the $x$-axis and the line joining $x$ and $y)$. For convenience, we let $N(x, c)=\{y \in X|| x-y \mid+a(x, y)<c\}$ and $D((x, y), r)$ denote the open disc centered at $(x, y)$ and with radius $r$.

It is easily seen that $(X, \tau)$ is stratifiable: For each open $U \subset X$ and positive integer $n$, let $U_{n}=U_{n}^{\prime} \cup U_{n}^{\prime \prime}$, where

$$
\begin{aligned}
& l_{n}^{\prime}=\bigcup\left\{D\left((x, y), \frac{1}{2 n}\right) \mid D\left((x, y), \frac{1}{n}\right) \subset U\right\}, \\
& l_{n}^{\prime \prime}=\bigcup\left\{N\left(x, \frac{1}{2 n}\right) \mid N\left(x, \frac{1}{n}\right) \subset U\right\} .
\end{aligned}
$$

A simple argument shows that $\left(U_{n}^{\prime \prime}\right)-\subset U$. Obviously, $\left(U_{n}^{\prime}\right)^{-} \subset U$, $\cup_{n=1}^{\infty} U_{n}=U$ and $U_{n} \subset V_{n}$ whenever $U \subset V$. Consequently $(X, \tau)$ is stratifiable (see footnote 2). Since $X$ is first countable then $X$ is a Nagata space.

However, Heath [4] proved that there exists no semimetric on $X$, which generates the topology of $X$, under which all spheres are open. As pointed out by Sims in [7], it follows that $X$ is not 1-continuously semimetrizable. (This is also easily seen by the use of Lemma 2.2.)

It is easily seen that $X$ is cosmic, since the subspaces $A$ $=\{(x, y) \in X \mid y=0\}$ and $Y=X-A$ of $X$ are separable metrizable spaces and thus $X$ is the continuous image of the disjoint topological union $A \vee Y$ of $A$ and $Y$.

EXAMPLE 2.4. There exists a cosmic (hence paracompact) 1-continuously semimetrizable space which is not stratifiable.

Proof. Let $X$ be the set of all points $(x, y)$ of the plane such that

(1) $y=0$ and $x, \sqrt{ } 2 / n-x, \sqrt{ } 2 / n+x$ are irrational for each positive integer $n$, or

(2) $x$ is rational and $y=\sqrt{ } 2 / n$ for some positive integer $n$.

A base for a topology $\tau$ on $X$ consists of all sets $B((x, y), n)$ $=\{(x, y)\} \cup\{(w, z) \in X|| w-x \mid<1 / n$ and $0 \leqq z-y<|w-x|\}$, (i.e. $B((x, y), n)$ is a "butterfly region centered at $(x, y)$ with radius $1 / n$ and vertex angle $\pi / 4 ")$ for any $(x, y) \in X$ and positive integer $n$.

Actually a "small" neighborhood of a point $(x, \sqrt{ } 2 / n) \in X$ is just an open interval of rational numbers in the horizontal line passing through $(x, \sqrt{ } 2 / n)$ and containing $(x, \sqrt{ } 2 / n)$. Also the only boundary points of a neighborhood $B((x, 0), n)$ are $(x+1 / n, 0)$ and $(x-1 / n, 0)$, 
since the hypotenuses of the "wings" of the butterfly $B((x, 0), n)$ contain no points $(w, \sqrt{ } 2 / n) \in X$ because of (1). Consequently one immediately sees that $(X, \tau)$ is a regular space.

Proceeding as in the last paragraph of the proof of Example 2.3 we can thus easily see that $X$ is a cosmic space.

Now we define a semimetric $d$ on $X \times X$ as follows:

$$
\begin{aligned}
d(x, y) & =|x-y| & & \text { if } a(x, y) \leqq \pi / 4, \\
& =|x-y|+a(x, y) & & \text { if } a(x, y)>\pi / 4
\end{aligned}
$$

where $a(x, y)$ denotes the smallest positive angle (expressed in radians) between the $x$-axis and the line joining $x$ and $y$ (we let $a(x, x)=0)$.

It is easily seen that $d$ is a semimetric on $X \times X$ which generates the topology on $X$. Furthermore one can easily show that $d$ is 1-continuous by the use of Lemma 2.2 and the crucial fact (as observed by the referee) that $a(x, y) \neq \pi / 4$ for all $x, y \in X$.

To complete the proof, we need only show that $X$ is not stratifiable. (We essentially use an argument which first appears in Heath [5]. However there are enough necessary changes which seem to warrant a complete proof.) Suppose that $X$ is stratifiable and $U \rightarrow\left\{U_{n}\right\}_{n=1}^{\infty}$ is a stratification of $X$. Let $X_{k j}=\left\{(x, 0) \in X \mid\right.$ and $B((x, 0), k) \subset U_{j}$ $\subset U \subset B((x, 0), 1)$ for some open $U \subset X\}$ for any positive integers $k$ and $j$. Clearly $\bigcup_{k, j=1}^{\infty} X_{k j}=\{(x, 0) \mid(x, 0) \in X\}$ and the real line minus the set of all $(x, 0) \in X$ is a countable set (it consists of rationals and one more irrational for each rational and each positive integer $n$ ). Therefore the closure of some $X_{m n}$ in the real line contains an open interval ] $a, b$ [, since the real line is of second category. Pick any $a<r<b$ such that $r+\sqrt{ } 2 / 2 m$ is rational (this is easily done!). Then $r$ is in the closure of $\left\{(x, 0) \in X_{m n} \mid x>r\right\}$ or $\left\{(x, 0) \in X_{m n} \mid x<r\right\}$; assume the former. Let $Q$ be the set of all $\left(U, U_{n}\right)$ such that

$$
B((x, 0), m) \subset U_{n} \subset U \subset B((x, 0), 1)
$$

for some $x \in X_{m n}$ with $r<x<r+\sqrt{ } 2 / m$. Then, letting $y=$ $(r+\sqrt{ } 2 / 2 m, \sqrt{ } 2 / 2 m) \in X$, we get that

$$
y \in\left(\cup\left\{U_{n} \mid\left(U, U_{n}\right) \in a\right\}\right)^{-}
$$

since $y \in\left\{U\left(B((x, 0), m) \mid x \in X_{m n}, \quad r<x<r+\sqrt{ } 2 / m\right\}\right)^{-}$because $1 / m>\sqrt{ } 2 / 2 m$. However,

$$
y \notin U\left\{U \mid\left(U, U_{n}\right) \in Q\right\}
$$

since $y \notin U\left\{B((x, 0), 1) \mid x \in X_{m n}, r<x<r+\sqrt{ } 2 / m\right\}$, a contradiction 
since we clearly have that

$$
\begin{aligned}
\left(U\left\{U_{n} \mid\left(U, U_{n}\right) \in Q\right\}\right)^{-} & \subset\left(U\left\{U \mid\left(U, U_{n}\right) \in Q\right\}\right)_{n}^{-} \\
& \subset U\left\{U \mid\left(U, U_{n}\right) \in Q\right\},
\end{aligned}
$$

by the assumption that $X$ is stratifiable.

Concluding remarks. By the symmetry of a semimetric, obviously a 1-continuous semimetric is 2-continuous and vice versa. Hence the semimetric $d$ of Example 2.4 is separately-continuous (i.e. continuous in each variable). However, it is not hard to see that $d$ is not continuous (consider any point $(x, 0) \in X$ and sequences $\left\{p_{n}\right\}_{n=1}^{\infty}\left\{q_{n}\right\}_{n=1}^{\infty}$ converging to $(x, 0)$ such that $a\left(p_{n}, q_{n}\right)>\pi / 4$ for each $n$-this can be easily done by choosing all $p_{n}$ and $q_{n}$ in the left wing of the butterfly $B((x, 0), n)$ with $p_{n}$ below $\left.q_{n}\right)$.

One might wonder then: Is every continuously semimetrizable space a Nagata space? Cook has answered that question. He has shown in [3, Theorems 7 and 8] that every continuously semimetrizable space is developable and that there exists a continuously semimetrizable space that is not normal and therefore necessarily not a Nagata space (or stratifiable).

\section{REFERENCES}

1. C. J. R. Borges, On stratifiable spaces, Pacific J. Math. 17 (1966), 1-16. MR 32 \#6409.

2. J. G. Ceder, Some generalizations of metric spaces, Pacific J. Math. 11 (1961), 105-125. MR 24 \#A1707.

3. H. Cook, Cartesian products and continuous semimetrics, Topology Conference at Arizona State University, Tempe, Ariz., 1967 (Editor: E. Grace).

4. R. W. Heath, A regular semimetric space for which there is no semimetric under which all spheres are open, Proc. Amer. Math. Soc. 12 (1961), 810-811. MR 23 \#A2862.

5. - A paracompact semimetric space which is not an $M_{3}$-space, Proc. Amer. Math. Soc. 17 (1966), 868-870. MR 33 \#3256. 1136.

6. J. L. Kelley, General topology, Van Nostrand, Princeton, N. J., 1955. MR 16,

7. B. T. Sims, Note on an example of Heath, Amer. Math. Monthly 70 (1963). 854-855.

University of California 